\title{
Collaboration Robots with Artificial Intelligence (AI) as Digital Doubles of Person for Communication in Public Life and Space
}

\author{
Evgeniy Bryndin \\ Scientific Department, Research Center "Estestvoinformatika”, Novosibirsk, Russia \\ Bryndin15@Yandex.Ru
}

\begin{abstract}
Trans-sectoral digital research of man, nature, society and industrial communication allows to create digital twins of social services. Digital duplicates associated with the service sector are created for intelligent process management. Digital twins of man provide services in the social sphere and in space. Training of digital twins in professional competences is carried out on the basis of communicative associative logic of technological thinking by cognitive methods. The study of the effectiveness of machine learning techniques, allowed the use of an approach that allows the combination of artificial intelligence and cognitive psychology. This approach provided pre-preparation of neural networks from accumulated data using existing behaviors. The approach combines existing scientific theories of human behavior with the flexibility of neural networks to make better decisions made by humans in space and in extreme situations. From a practical point of view, this makes it possible to more accurately determine the behavior of the human digital twin in space and in extreme situations. There are a number of socio-economic issues related to human-machine interaction. Complex technologies are not credible on the part of citizens. The coming years will take to improve safety and standardize the creation, application of digital twins and behavior of robots.
\end{abstract}

Keywords: technological thinking; communicative and associative logic; digital doubles; communicative associative logic; adaptive behaviour

\section{Introduction}

The term Digital Twins appeared in the early 2000s, but every year, as technology developed, it received new content. The basic concept is not difficult to understand: monitoring of a physical object is carried out on the basis of a closed cycle of information exchange between it and its virtual model (thus a digital twin).

A digital twin is a virtual prototype of a real object, group of objects, or processes. It is a complex software product that is built from a wide variety of data. The digital twin is not limited to collecting data obtained during the development and manufacture phase of the product - it continues to collect and analyze data during the entire life cycle of the real object, including through numerous IoT sensors. A digital twin is a virtual reproduction of the operational state of a real physical object, process, system, or whole service. It can be a virtual twin of a part, product, equipment, process, production sites, workshops, or even factories. It is essentially a set of mathematical models describing the state of an object and all its elements. In general, the digital twin includes: geometric model of the object; set of calculated data of parts, nodes and the object as a whole (Mathematical models describing all physical processes taking place in the object); information on technological processes of manufacturing and assembly of individual elements; some data on tests of the object, for example, readings of sensors, from which calculated data can be confirmed; system of product life cycle management, which links all the above objects into a single structure.

The digital twin is used in association with the physical throughout the life cycle: during the testing, rework, operation and disposal phase. The physical object uses sensors that collect real-time data about the state of the object, after which this information is sent to the digital 
twin. On the basis of the obtained data, the digital model is clarified, which, in turn, gives recommendations for optimization of the operation and maintenance mode of the real object.

The digital twin allows you to simulate in virtual space the change in the state and characteristics of the entire product when the characteristics of any of its elements change. Its main task is to allow real-time management of all factors affecting the cost and quality of the product even before its production begins.

Digital twins are created to significantly accelerate the time to market of new products, so you can present all stages of its life cycle in a virtual environment. Another function of the digital twin is to inherit product data when modifications are made to it. In other words, to maximize the use of previous experience in designing, manufacturing, and testing new product modifications. In this case, we take into account the peculiarities of the product operation and on the basis of the data obtained from the digital twin, we can improve the characteristics of the product modification.

The main advantage is the speed of making technical decisions and the cost of obtaining the required characteristics of the product. Having a digital twin, it is possible to reduce by an order of magnitude the number of natural tests, the number of attempts to work out technological processes, all that is connected with the production of a real material part and its tests, the cost of which is much higher than the cost of mathematical modeling.

Another advantage is the possibility of collective work on the product of geographically remote collectives and engineering centers. At the same time, the necessary scientific potential and labor resources can be used than with manual design technology.

If we consider the digital twin not of a specific product, but of the whole production, the advantage is the possibility to simulate in a virtual environment all processes, to determine the necessary quantity and optimal location of equipment depending on the volume and range of products produced. At the same time, if a digital twin is developed for the newly created production, it is possible to identify possible risks and shortcomings through simulation of its work, to correct the project. The digital twin of existing production allows you to work out the implementation or change of technological processes without real interference with the work.

Digital twins allow you to model a variety of situations that can occur in production. Thus, the digital twin allows to select the most adequate scenarios of technological processes to avoid failures and force majeure. Digital twins help improve the efficiency of the cloud platform, solve design problems early, train employees, support innovation, and more.

Digital twins are starting to use companies of different industries, such as energy, transport, construction, but first of all it works in high-tech industry (Bryndin, 2019), (Bryndin, 2016), (Bryndin, 2017). The most dynamic market where digital twins are used is the global automotive industry, which produces 100 million units annually.

\section{Review of Literature}

\subsection{Artificial intelligence methods, systems and techniques}

Big Data. The data collected automatically is too large for manual collection and processing to find new patterns and knowledge that cannot be derived from local data fragments.

Recommendation Systems (Collaborative Filtering) are systems that provide recommendations based on implicit patterns detected by AI algorithms using big data analysis. 
They can be aimed at interaction with the client (offer goods/services/dating), or used within companies (to support credit decisions, personnel decisions).

Predictive systems are systems that capture relationships between variables in historical data sets and their outcomes. Based on these relationships, models are developed, which in turn are used to predict new results.

Biometrics - removal and digitization of various biological characteristics of a person faces, gestures, gait, fingerprints, blood pressure, temperature, etc. Used for identification of people and human-machine interactions.

Knowledge Management is the representation of knowledge about the world in the form of a hierarchy of concepts, such that the AI system can use knowledge to solve complex problems such as disease diagnosis, decision making, or human conversation.

Man-Machine Interface. Person-Device Dialog - A dialog (text or voice) in a client or technical support service. Entertainment robots. Dialogue with household appliances, car, disabled.

Virtual interlocutors (chatbota, Chatbots) - the programs communicating with the person in a natural language. Can work as promoters, account managers, help human resources, answer frequent questions in online technical support services, use in cars and home entertainment robots. Communication can be text (chat-like) or voice.

Machine Learning (ML) is an AI area that creates algorithms that independently learn and predict the situation based on available data without requiring the intervention of a programmer.

Supervised Learning is a type of machine learning where the learning algorithm is given pre-classified and sorted data consisting of examples of input data and desired results of its processing. The purpose of the training is to learn the general rules that link input and results and, based on these rules, to predict future events.

Unsupervised Learning is a kind of machine learning in which the learning algorithm does not include any classifications and labels, itself defines the structure and relationships of the input data. Uncontrolled learning can itself be a goal (detection of hidden patterns in data) or a means (extraction of features from an array of data). It is focused on the investigation of input data and recognition of the structure of untagged data.

Reinforcement Learning is a type of machine learning in which an algorithm has a specific purpose - for example, to control a robotic manipulator or play Go. Every step on the way to the goal is marked by a reward or a fine. Given this feedback, the algorithm can work out the most efficient path to the target.

Deep Neural Networks is a multi-layer neural network in which "learned" data is passed through several layers of "neurons"; On each layer, the result of the previous layer is used as input information.

Convolutional Neural Networks. Multilayer neural networks with alternation of special curving (summing) and ordinary layers, most effective for image recognition.

Feed-Forward Networks is a neural network with many layers where data is propagated only forward.

Recurent Neural Networks are deep neural networks where data can propagate between layers forward and backward.

Neural Network Constructors/Libraries (Frameworks/Neural Frameworks, Deep Learning Frameworks). Neural network development environment "under task," allowing generate several dozen different types of neural networks and then train them on given 
datasets. There are only a few popular neural libraries, the main players of this market TensorFlow (Google), PyTorch (Facebook), Keras (open-source), etc.

Graphics accelerators (Graphics Processor Unit (GPU)). Graphic maps for displaying images on the computer screen, allowing to carry out ultra-fast and massive parallel calculations, due to this became the main carrier of calculations on neural networks. The main player of this market is Nvidia.

Neural chips (Neural Processing Unit, Neuromorphic Processor, Tensor Processing Unit, ASIC). Highly specialized processors specifically optimized for fast parallel computing using neural networks.

Dataset (Training Set) a set of precedents (objects, cases, events, subjects, texts, photographs, samples, etc.) selected to train an algorithm from a variety of all possible precedents, called a general set.

Mark-up. Processing a learning sample to assign properties to objects in it that the algorithm is learning to recognize.

Marking system. An automated workplace that allows many machine learning operators to quickly map learning samples. For example, manual allocation of persons by the operator in photos, marking of letters on spam/non-spam, marking of search results or X-rays.

Open data - archives, libraries of data, usually stored in cloud storage and available for download anywhere in the world to anyone.

Data Processing Center, Data Center - a special technical room, which houses special equipment (servers, server racks, network equipment, etc.) for processing, storage and transmission of large data arrays, with power supply and powerful communication channels. Modern data centers are complex engineering facilities and consume a large amount of electricity (up to hundreds of megawatts), most of which goes to cooling the equipment.

The data set (data set) represents a set of the data having identical structure and united on sense. The dataset can be both text, sound, visual and tabular information and all kinds of combinations thereof. The dataset may contain data annotated (marked) or unannotated (unmarked).

Tagged data - containing structured information for all dataset instances that include meta-information to solve specific applications. The markup shall be unified, have the property of integrity and meet the requirements of meta content.

Julia is a high-level general-purpose programming language developed in 2009 with a focus on high-performance numerical analysis and computational science. Simple syntax and deep mathematical roots make Julia a friendly programming language for data analysts, offering the perfect way to express algorithms. It also includes Flux, the basis for machine learning and supports TensorFlow and MXNet.

Python is a widely used programming language and can be used to implement AI because of the simple and seamless structure it offers. Python syntax makes it easy to implement different AI algorithms, which also reduces development time compared to other available programming languages. Applying Python allows users to create neural networks with a set of useful libraries that can be used to develop AI. Other features include the ability to test algorithms without having to implement them.

Java, an extremely popular cross-platform programming language, provides search algorithms and neural networks. It is an easy-to-understand language that offers graphical representation, debugging, and scalability. Its portability makes it the preferred implementation for different applications based on having different built-in types. 
Tentorflow is an open software library for machine learning developed by Google to solve the problems of building and training a neural network with the aim of automatically finding and classifying images, achieving the quality of human perception. It is used for both research and development of Google 's own products. The main API for working with the library is implemented for Python, and there are also implementations for C, Haskell, Java, Go, and Swift. Continues a closed DistBelief project. Originally TensorFlow by the Google Brain team for internal use at Google, in 2015 the system was transferred to free access with an open license Apache 2.0. TensorFlow is well suited for automated image annotation in systems such as DeepDream. TensorFlow allows training of GANs.

PyTorch is an open-source Python machine learning library built on Torch. Used for natural language processing. It is developed predominantly by Facebook 's artificial intelligence group. Uber 's "Pyro" library for probabilistic programming is based on PyTorch. PyTorch provides two main high-level models: 1. Tensor computing (similar to NumPy) with advanced acceleration support on GPU, 2. Deep neural networks based on autodiff system.

Keras is an Open Neural Network Library written in Python. It is an add-on above the Deeplearning4j, TensorFlow and Theano frames. It is aimed at operational work with deep learning networks, while being designed to be compact, modular and expandable. According to the concept, Keras is an interface rather than an end-to-end machine learning system. Keras provides a high-level, more intuitive set of abstractions that makes it easy to form neural networks, regardless of the scientific computing library used as a computational backend. Microsoft is working on adding CNTK libraries to Keras and low-level libraries.

Caffe is the foundation of deep learning, created with speed and modularity in mind. Switching between the central and graphic processing unit on the computer, and then expansion on computing clusters or mobile ustroi• stvo. Speed makes Caffe ideal for scientific experiments and industry. Caffe can process 60 million the image in day with one GPU NVIDIA.

CUDA is a parallel hardware and software architecture that significantly improves computing performance with Nvidia graphics processors. The CUDA SDK allows programmers to implement algorithms in special simplified dialects of the $\mathrm{C}, \mathrm{C}$ and Fortran programming languages that are feasible on Nvidia graphics and tensor processors. The CUDA architecture gives the developer the option, at his own discretion, to organize access to the set of instructions of the graphic or tensor accelerator and to manage its memory. Functions accelerated with CUDA can be called from a variety of languages, including Python, MATLAB, etc.

Clouds - computing services (servers, storage, network, software) accessible via the Internet. In fact, you can buy computing power or storage without thinking about what servers and where they are - somewhere "in the cloud." For example, to avoid storing personal documents and photos on a personal computer 's hard drive, most users now store them online.

Artificial Intelligence (AI) is a scientific applied direction for the development and creation of technological and program cognitive complexes of the digital twin of human intelligence, capable of learning, retraining, self-realization and development on the basis of the criterion of preferences and to improve functional activity by qualitative selection and mastering of creative innovative high-tech professional and behavioural skills and competences.

First of all, these are numerous methods of modeling processes (physical and information), which both assess its current work and allow make forecasts for the future: how 
the model will behave when changing its parameter. Other methods allow you to study the external parameters and structure of an object, analyze properties, or examine existing parameters and running processes. The third is to obtain data and reliability of the object or system obtained on the basis of a model analyzing the consequences of risks. At the same time, all data can be obtained together and analyzed in a complex.

It is also necessary to build accurate analytical models that can be applied to the digital twins. By digital twin is meant a set of digital technologies that use approaches of statistical analysis, machine learning, chemistry, physics, control theory, reliability theory, mass service theory, numerical modeling, optimization, simulation modeling.

Solutions using digital twins are built on a whole set of technologies.

Digital Twin Aggregate, DTA (Aggregated Twin), is a standard computing system that combines all digital twins and their actual prototypes, allowing data to be collected and exchanged. DTA twins are defined as a computing system that has access to all digital twin instances and can send them requests in random or active polling mode.

The digital twin allows reproduce all other indicators of the object by minimum key parameters. With this technology it is possible to solve various classes of tasks of diagnostics of object state, forecasting, optimization of operation, control.

The digital model also contains a history of maintenance and operation. Taken together, all of this data makes it possible to predict the behavior of a real object. In addition, it is possible to monitor and test the entire fleet of facilities and carry out analysis on the basis of aggregated data.

It is important to note that machine learning technologies are also involved in digital twins, Because they are essentially self-learning systems that use information from a range of sources, including data from sensors monitoring various performance indicators of the physical object, Information from expert experts and larger systems, of which the observed physical object may be a part.

Digital twins can also be created for business processes. Automation methods allow to model the optimal path of development.

Digital twins have become a really strong catalyst for the development. Robots as human digital twins (Markets and Markets Research, 2017), (Robotics Business Review, 2017), (Cision, 2017), (Gizmodo, 2017) significantly save resources, minimize risks of errors and failures, which prolongs the period of stable operation. All this allows to get the maximum possible return on investments, increase competitiveness and increase demand.

\section{Discussion}

\subsection{The adaptive behavior digital robots}

Today there is a set of algorithms of machine learning on the basis of which it is possible to construct scoring model: Decision Tree (decision tree), KNN (method of the k-closest neighbors), SVM (method of basic vectors), NN (neuronet). And the choice of model should be based on what we want from it. First, as far as the decisions which affected results of model have to be clear. In other words, as far as it is important to us to have an opportunity to interpret structure of model of adaptive behavior. They give the chance very deeply and seriously to configure architecture and settings of training (Wermter et al, 2005), (Bryndin, 2018), (Bryndin, 2017), (Domingos, 2016), (Bryndin, 2019), (Artificial Intelligence \& Robotics, 2019), (Zhang et al, 2018), (Bryndin, 2019). 
Very soon the need for call center services will disappear, and no longer have to wait. According to a study conducted by Gartner, artificial intelligence will perform 85 percent of customer service work by 2020 . In principle, this was to be expected, as Facebook and other social networks successfully use chat bots to process calls to support. When artificial intelligence takes matters seriously, waiting times for various transactions will be significantly reduced, which is an obvious benefit for companies and their clients.

Gartner also reports that artificial intelligence will "mimic human communication by learning to listen and speak, remember what has been heard, take into account the situation, time and tone of the conversation. Robots will maintain communication, develop your thoughts and suggestions about various accidents and objects. " In other words, an electronic assistant will be able to recognize a person closer, and a conversation with a cyborg will resemble a friendly conversation or something like that.

By 2018, artificial intelligence will create 20 percent of business content. Artificial intelligence will significantly affect the economy. The Bank of America, in a report to investors, indicated that the introduction of artificial intelligence would affect the economy in the form of a recession in some of its areas, with substantial growth in others. Overall, this "creative destruction" is estimated at \$14-33 trillion. According to experts, the process is just beginning. Investing in these technologies is guaranteed to generate income.

\subsection{Specialization of cognitive adaptive professional robot}

The functional structure of specialization of the cognitive adaptive professional robot with retraining consists of various systems with artificial intelligence (Figure 1).

Specialization of cognitive adaptive robots is carried out on the basis of knowledge bases, bases of abilities and implementers of behavior. Cognitive adaptive robots with imitative thinking and adaptive behavior have prospect of broad practical application.

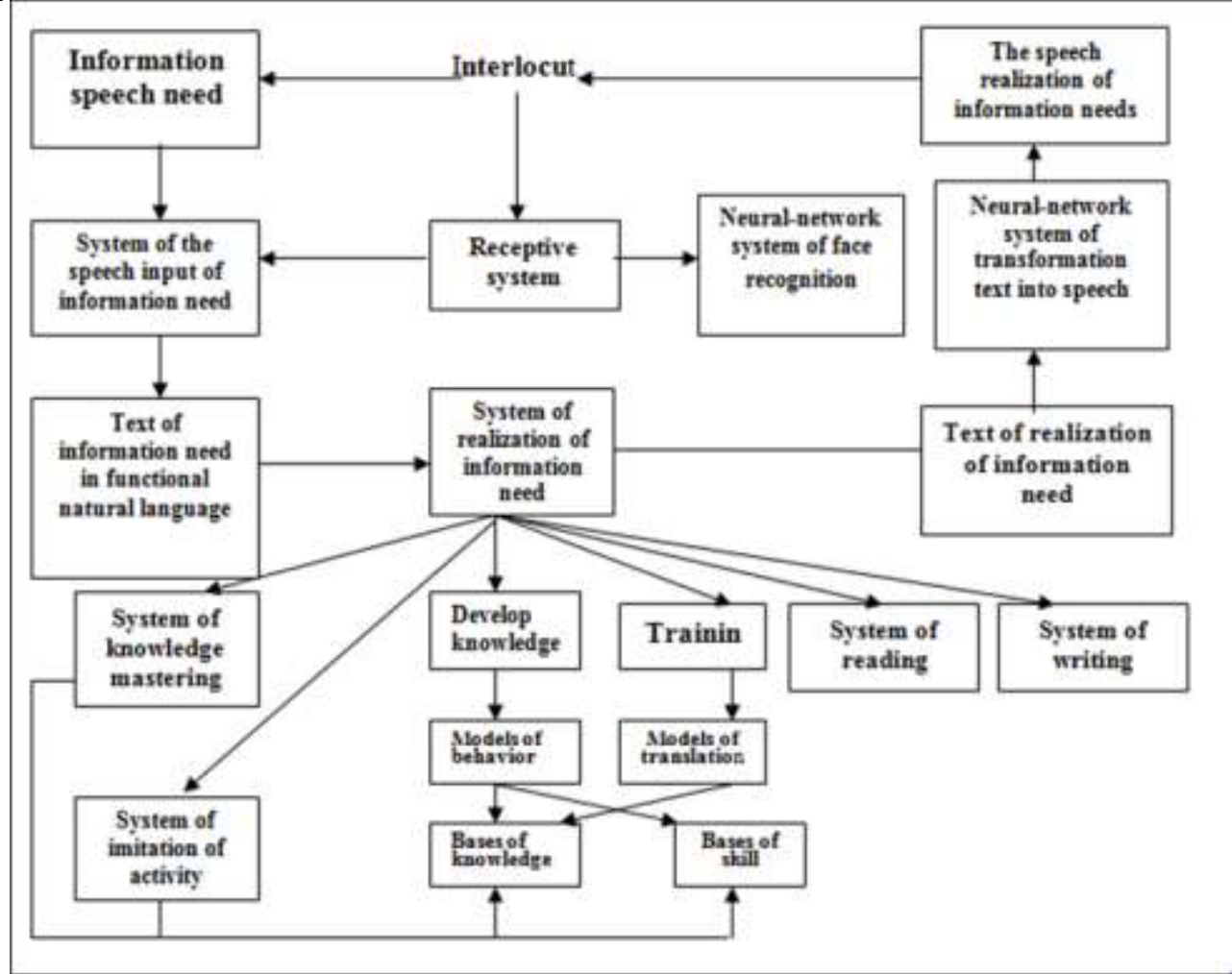

Figure 1. Functional structure of the cognitive professional digital doubles 
Budapest International Research in Exact Sciences (BirEx) Journal

Volume 1, No 4, October 2019, Page: 1-11 e-ISSN: 2655-7827 (Online), p-ISSN: 2655-7835 (Print) www.bircu-journal.com/index.php/birex emails: birex.journal@gmail.com birex.journal.qa@gmail.com

Technological cognitive thinking of the robot is carried out on the basis of an integrated approach: linguistic, statistical, psychological and others (Samuel, 2019), (Rad, 2019).

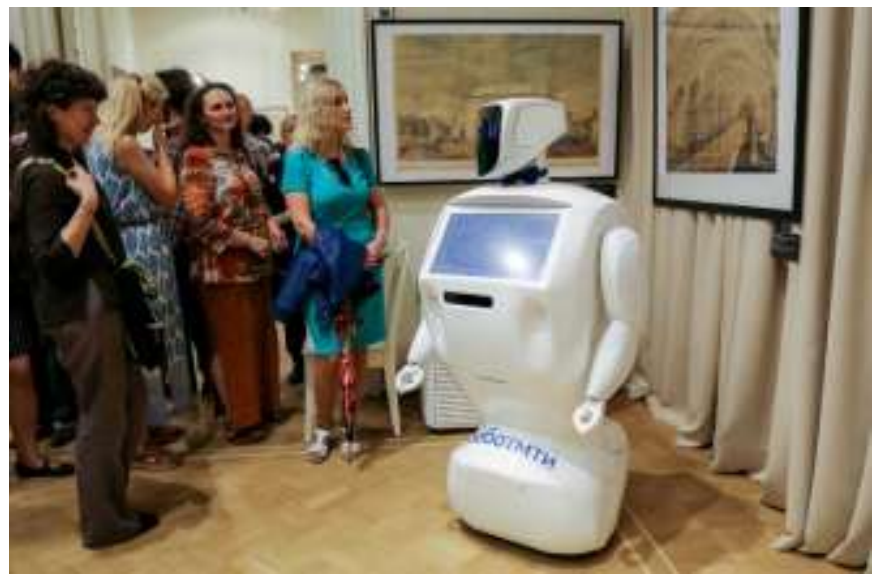

Figure 2. Russian robot guide in art gallery

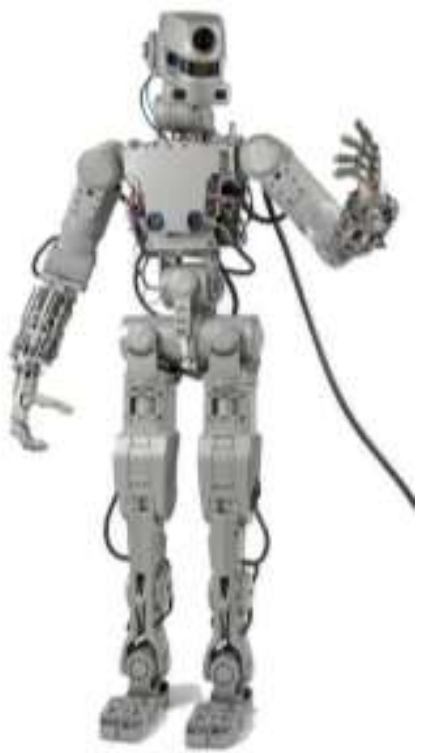

Figure 3. Russian robot astronaut FEDOR for work in space

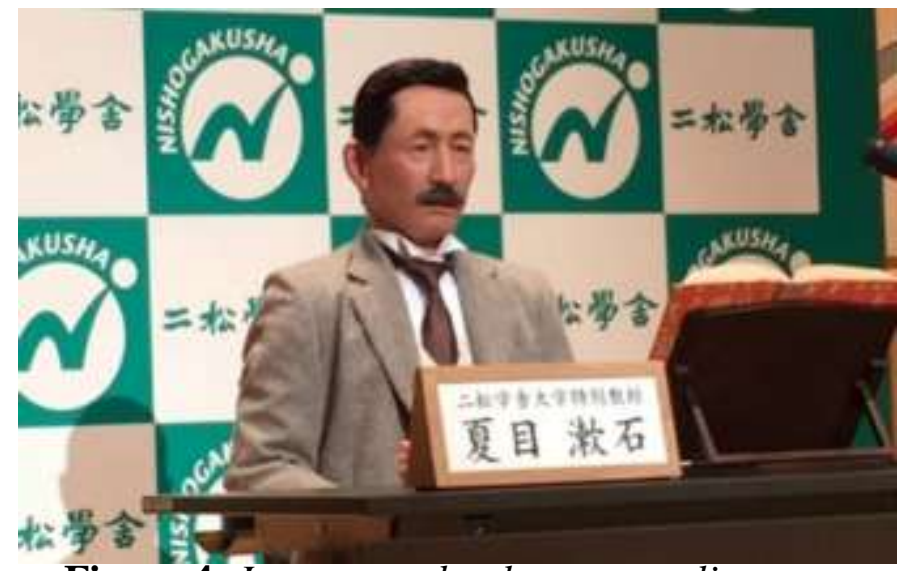

Figure 4. Japanese robot lecturer on literature 


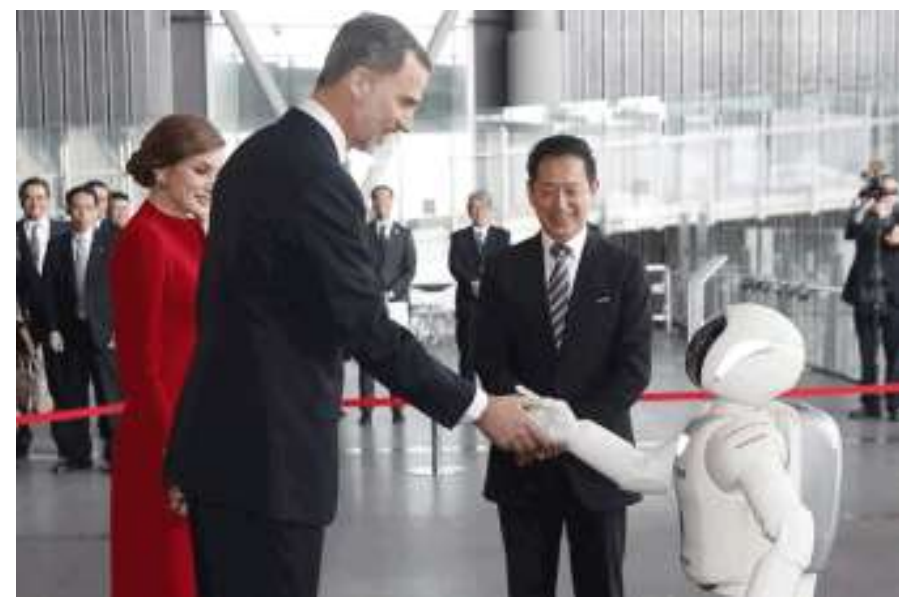

Figure 5. Japanese robot-Android volunteer ASIMO

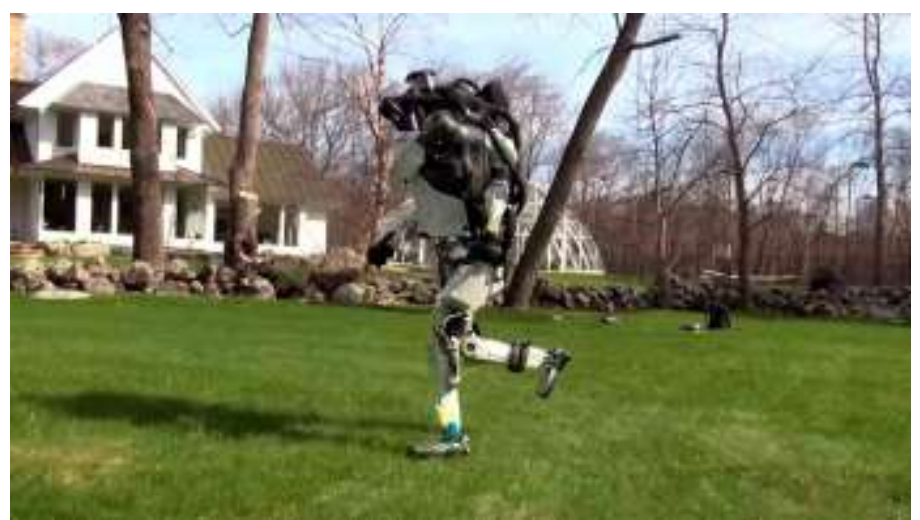

Figure 6. American mobile robot security guard Atlas with adaptive behavior

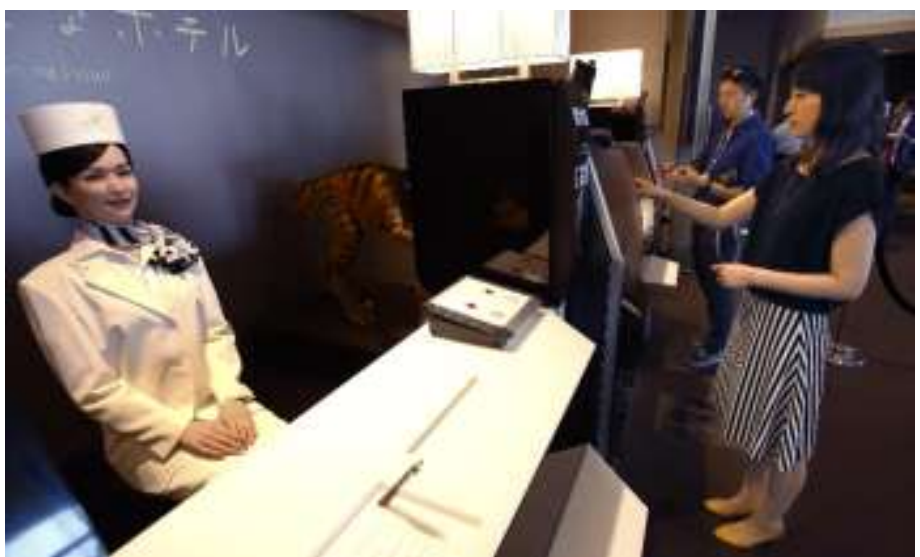

Figure 7. Japanese robot-administrator of hotel Henn-na Hotel.

\section{Conclusion}

The cognitive adaptive professional robots with communicative and associative logic of thinking having the systems of machine retraining of realization of information requirements will be able quickly to change professional qualification and competences. The smart cognitive architecture of the robot using criterion of improvement of functional activity is 
capable to recurrent self-improvement. The cognitive architecture includes artificial neural networks, algorithms of machine learning, the cognitive smart big data system, the system of high-quality selection. The smart cognitive architecture of the robot step by step defines how it is the best of all to achieve the set objectives and to realize preferences by means of actions of function of usefulness on the basis of high-quality selection. Self-improvement is carried out by machine retraining by criterion of preferences on the basis of extensive statistics of high-quality selection of skills and competences. The smart cognitive architecture of the robot seeks for development of all aspects of artificial intelligence by machine retraining, on the basis of extensive statistics of the acquired professional and behavioural skills.

The international scientific and engineering society gradually moves to technical realization of the cognitive professional robot with retraining. The group of the University of California in Berkeley and Princeton investigating efficiency of methods of machine learning for forecasting of human behavior offered the new approach which is given rise on a joint of AI and cognitive psychology. Scientists presented the new concept providing preliminary training of neural networks at the synthetic data prepared by psychologists by means of the existing theoretical models. Approach can be used by other groups for a training of their own models of machine learning. Approach combines the existing scientific theories of behavior of the person with flexibility of neural networks for the best forecasting of the risky decisions made by the person. From the practical point of view it allows to save to researchers a lot of time which is spent usually on data collection for the knowledge base of human behavior.

The automated system of retraining can retrain the cognitive robot on other specialization, described in article. The expert has to prepare the knowledge base, base of abilities, behavior model and model of the environment. Degree of accuracy of the description of the external environment, behavior model and realization of information requirement defines correctness of performance of human tasks by robot.

In the future in labor market cognitive robots with retraining will perform professional works, and the person will occupy a niche of scientific research of creative innovative activity.

Robots become independent subjects of social environment. Social cognitive smart robots are used as guide, seller, lecturer, vacuum cleaner, nurse, volunteer, security guard, administrator of hotel, astronaut.

\section{References}

Bryndin, Evgeniy. (2019). Human Digital Doubles with Technological Cognitive Thinking and Adaptive Behaviour. Software Engineering, 1-9.

Bryndin, Evgeniy. (2016). Cognitive robot consultant for a healthy lifestyle. III International scientific conference "Information Technologies in Science, Management, the Social Sphere and Medicine", 484-488.

Bryndin, Evgeniy. (2017). Cognitive Robots with Imitative Thinking for Digital Libraries, Banks, Universities and Smart Factories. International Journal of Management and Fuzzy Systems, 57- 66.

Markets and Markets Research "Collaborative Robots Market", 2017. URL: https://www.marketsandmarkets.com/Market-Reports/collaborative-robot-market194541294. html 
Robotics Business Review — “The 2017 RBR50 List Names Robotics Industry Leaders, Innovators”, 2017 URL: https://www.roboticsbusinessreview.com/download/2017rbr50-list-names-robotics-industry-leaders-innovators/

Cision. (2017). "Top Robotics Market by Industrial Robotics, Service Robotics - Global Forecast to 2022". URL: https://www.prnewswire.com/news-releases/toproboticsmarket-by-industrial-robotics-service-robotics---global-forecastto-2022300405291.html

Gizmodo. (2017). "Robots Are Already Replacing Human Workers at an Alarming Rate". URL: https://gizmodo.com/robots-arealready-replacing-human-workers-at-an-alarmi1793718198

Wermter, S., Palm, G., \& Elshaw, M. (2005). Biomimetic Neural Learning for Intelligent Robots: Intelligent Systems, Cognitive Robotics, and Neuroscience. Springer-Verlag Berlin Heidelberg.

Bryndin, Evgeniy. (2018). Technological Thinking, Communication and Behavior of Androids. Communications, 13-19.

Bryndin, Evgeniy. (2017). Program Hierarchical Realization of Adaptation Behavior of the Cognitive Mobile Robot with Imitative Thinking. International Journal of Engineering Management., 74-79.

Domingos, Pedro. (2016). Supreme algorithm. How machine learning will change our world. M.: Mann, Ivanov and Ferber.

Bryndin, Evgeniy. Social Cognitive Smart Robots: Guide, Seller, Lecturer, Vacuum Cleaner, Nurse, Volunteer, Security Guard, Administrator. Communications, 6-12.

Artificial Intelligence \& Robotics: Industry Report \& Investment Case. (2019). https://indexes.nasdaqomx.com/docs/NQROBO\%20Research.pdf

Zhang, Shuai et al. (2018). Deep Learning based Recommender System: A Survey and New Perspectives,. https://arxiv.org/pdf/1707.07435.pdf

Bryndin, Evgeniy. (2019). System retraining to professional competences of cognitive robots on basis of communicative associative logic of technological thinking. International Robotics Automation Journal, 112-119.

Google's Artificial Intelligence Built an AI That Outperforms Any Made by Humans. (2019). https://futur-ism.com/google-artificial-intelligence-built-ai

Samuel, A, F. (2019). On the Performance of Transmuted Logistic Distribution: Statistical Properties and Application. Budapest International Research in Exact Sciences (BirExJournal), 26-34.

Rad, Mostafa Rahimi. (2019). Learning Discourse Analysis by Applying MetaphoricallyBased Materials. Britain International of Linguistics, Arts and Education, 9-17. 